\title{
Biospecimen quality program in the biobank of the Norwegian Institute of Public Health
}

\author{
Liv Paltiel, Jeanette Aarem, Siri Bækken, Nina K. Stensrud and Kari Harbak \\ Norwegian Institute of Public Health, Division of Public Relations and Institute Resources, \\ Department of Biobank, Oslo, Norway \\ Correspondence: Liv Paltiel, The Norwegian Institute of Public Health, Division of Public Relations and Institute Resources, \\ Department of Biobank, P.O. Box 4404 Nydalen, NO-0403 Oslo, Norway \\ E-mail: liv.paltiel@fhi.no Telephone: +47-21076716
}

\begin{abstract}
Background: The biological material collected, processed and stored in biobanks are important research tools and it is important to minimize preanalytical variations to provide researchers with high quality biological material that will give reproducible results.

Methods: To minimize the preanalytical variations caused by sample collection, processing and storage, we have established a biospecimen quality program. It consists of quality assurance aspects as well as quality control programs to measure adherence to protocols and sample integrity. The quality control program includes measurements and evaluation of the DNA quality and quantity before storage, i.e. concentration, purity, fragmentation and PCR success, and long term storage programs for plasma, urine and RNA.

Conclusions: The Biobank at the Norwegian Institute of Public Health has established a biospecimen quality program that ensures high quality specimens and provides the documentation required to use the biomaterial in a best possible way.
\end{abstract}

\section{INTRODUCTION}

Human biobanks are important research tools for studying factors contributing to disease, and research using samples from biobanks is dependent on both high quality samples and associated data. Thus, it is essential that biospecimen handling, i.e. collection, processing, storage and retrieval, is conducted under quality assurance (QA) and quality control (QC) programs. A QA program will help minimize preanalytical variations which may affect the stability of target biomarkers. Preanalytical variations are defined as any variation taking place between the moment of specimen collection and the moment of sample analysis [1], and it is previously reported that pre-analytical errors count for 31$75 \%$ of all errors in laboratory medicine [2]. Clear and explicit standard operating procedures (SOPs) must be developed and implemented at the biorepository, and adherence to these SOPs is a necessity as it decreases the variance in inter-sample analytical properties [3], and allows for comparability of the biological samples. In addition, biospecimen QC programs should be conducted to measure adherence to the established SOPs and the impact of different preanalytical variables on the integrity of the biological samples. Data from such programs will allow for optimization of SOPs and will clarify the realistic use of the stored biospecimen in future research and analysis.

The Biobank of the Norwegian Institute of Public Health (NIPH) has grown to become a state of the art unit for long-term storage and processing of biological samples used in national and international research. It was established to process and store the more than 350,000 sample sets in the Norwegian Mother and Child Cohort Study (MoBa) [4,5], but can now offer its large storage and processing facilities to other institutes and companies in addition to internal projects. Today the NIPH Biobank houses samples from more than 380,000 human donors and with many samples divided into aliquots, the number of storage units is close to 5 million. Most of the samples are whole blood, plasma, DNA and urine, stored in 96 well tray formats labelled with standard barcode or 2-D barcode. As of June 01, 2011, about 30000 aliquots have been retrieved from the Biobank and used in research projects in various research areas including preterm birth, preeclampsia, autism, eating disorders, multiple sclerosis and twin studies.

To ensure good quality of both the biological materials and administrative processes, a quality management system based on NS-EN ISO 9001:2008 and quality programs for the different types of biomaterial have been developed and implemented.

The quality management system (QMS) covers all activities of the Biobank and ensures the quality of the biomaterial and fit-for-purpose processes. It includes a quality policy, customer focus and contact, resource management, document handling, processes and procedures for product realization and control, internal audits and continuous improvement, which is one of the pillars in the NS-EN ISO 9001: 2008 standard. Thus, the QMS ensures usage of findings and results from all our activities, including our biospecimen quality program, to improve our processes and quality. 
Biobanking is an investment for the future and for most studies where biological material is stored in the NIPH Biobank, the specific analyses of the biomaterial have not been determined at the time of sample collection, handling and storage. To be able to provide researchers today, and maybe decades from now, with the best possible foundation for their decision on how to best use the material in the Biobank, we conduct long-term storage studies to monitor the sample's behaviour. In addition pre-analytical factors are tracked in our LIMS and we keep all instructions for collecting and shipping samples, as well as all old versions of SOPs describing the sample processing for future references. Every time a new project is planned, or we receive a request for our biobanking services, we make a project specific QC program for the biological material, to assure traceability, sample quality and best practices for the purpose of the project within the available funding.

In this manuscript we present the quality programs we have developed and implemented for the different types of biological material stored in the NIPH Biobank.

\section{Methods}

\section{DNA}

Extraction of DNA from whole blood is considered the "gold standard" for downstream applications like whole genome amplification and high density SNParrays, applications that demand high quality DNA [6]. At the Biobank, DNA is manually extracted from whole blood using commercial kits. The sampling and sample processing at the Biobank is described in detail elsewhere [5].

Quality control of DNA should consist of both analytical and functional QC [7]. At the NIPH Biobank we have chosen to include concentration, purity, fragmentation and PCR success in the DNA QC program, Figure 1. In addition we also check each batch from the DNA isolation process for contamination when measuring OD for the negative controls in the DNA normalization process [5].

As a part of the normalization process, purity is assessed by the OD 260/280 ratio for all samples using Spectramax 190 spectrophotometer (Molecular Devices), and the ratio has to be 1.6-2.0 [5]. Samples with a ratio $>2.0$, which may indicate RNA contamination [8], will be included in the Biobank, but with a remark in the database stating that the sample is outside the purity requirement including the measured ratio. Samples with a ratio $<1.6$ will go through a new DNA purification process up to two times to remove any remaining protein contamination, and are set aside for future purification if the ratio still is $<1.6$.

To facilitate DNA sample delivery DNA concentration is normalized to $100 \mathrm{ng} / \mu \mathrm{l}$ [5], and as part of the QC program the concentration after normalization is measured for every $12^{\text {th }}$ sample. Every week the mean and standard deviation are calculated. The requirement for the mean is $95-105 \mathrm{ng} / \mu \mathrm{l}$, and the standard deviation $\leq 15 \%$. For the measured samples it is remarked in the database if they have concentrations $<70$ or $>$ $130 \mathrm{ng} / \mu \mathrm{l}$, corresponding to the $\max 2 \mathrm{SD}$ for the process. If more than one of 8 samples are outside the same interval, an evaluation is done whether the normalization, aliqouting and storage process should be temporarily stopped to seek any error in the sample handling or instruments used, and to find a solution to get the process within the chosen requirements. In addition we conduct a weekly evaluation of the results where the concentration trend is considered as well as possible differences between our 5 liquid handling robots and the 8 tips of each robot.

Every 3 months, 48 randomly selected DNA samples go through the fragmentation and PCR success parts of the QC program. The extent of fragmentation is evaluated on a $0.8 \%$ agarose E-gel (Invitrogen) containing ethidium bromide. HindIII digest of $\lambda$ DNA (Invitrogen) is used as a size marker and the bands are visualised by ultraviolet illumination. Samples with fragmentation will be remarked in the database and an evaluation of both the DNA isolation procedure and normalization process is done if more than one of 48 samples show fragmentation.

As an indication of PCR inhibitors and DNA fragmentation, the ability to amplify a $1.2 \mathrm{~kb}$ fragment of a housekeeping gene (PLAT) is measured by Real-Time PCR (PLAT13Fw: 5'-CAAATGAGATCCCGCCTCT TC-3', PLAT13Rv: 5'-GAGCCCCCATAATCTAAA TTCCA-3' (MedProbe) PLAT13Probe: 5'- TCCAGA CCCACCACACCGCAGA-3' (Applied Biosystems)). $\mathrm{A} \mathrm{C}_{\mathrm{t}}$-value $<27$ is regarded as a positive result. Whenever the result is regarded negative, i.e. there is no product or the $\mathrm{C}_{t}>27$, the PCR will be repeated once. If the result still is regarded negative after one re-run, the sample is remarked in the database and an evaluation of the DNA isolation and normalization processes is conducted.

\section{Plasma}

Not knowing what analyses would be performed on the EDTA-plasma in MoBa, a program to assess the sample integrity during storage at $-80{ }^{\circ} \mathrm{C}$ was established. A quality assurance pool was created and a selection of plasma analytes was chosen to measure changes not only during long-term storage, but also during repeated freeze-thaw cycles [9].

The long term storage part of the program will continue as long as we have plasma samples. Concentrations of sodium, free fatty acids, AST, cholesterol, triglycerides and vitamin $\mathrm{E}$ have been measured once a year the last 6 years, and will be checked regularly also in the years to come. The analytes were chosen to represent different aspects of sample integrity [9]. Any statistical change will be reported to the PI and/or steering groups of the $\mathrm{MoBa}$ and other projects storing plasma in the Biobank. 


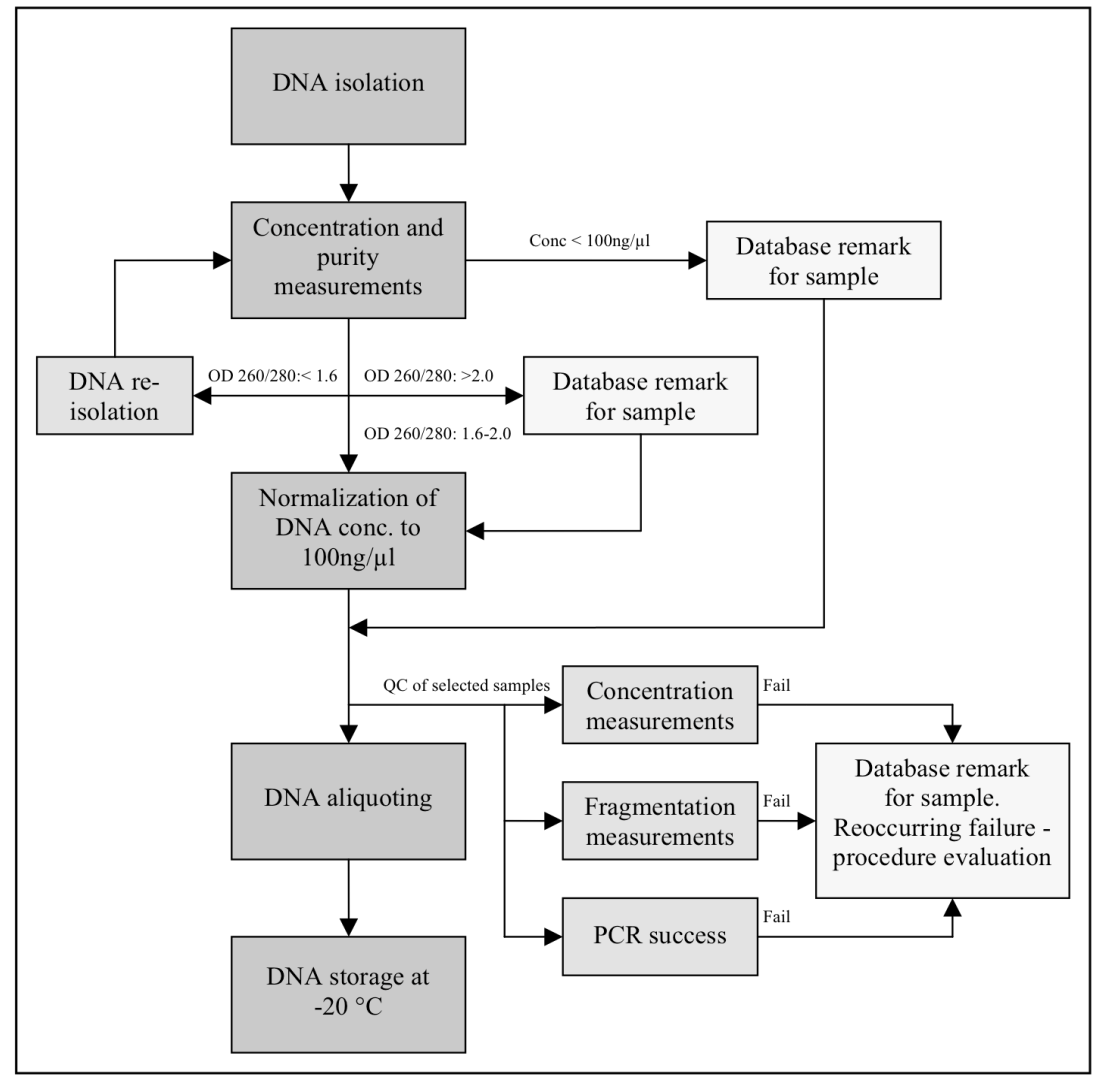

Figure 1. Flow chart of the DNA processing and quality control program.

\section{Urine}

We have started a program to follow the concentration of two markers, sodium and creatinine, in urine during long-term storage [5]. Sodium was chosen as a marker of volume and creatinine was chosen because many investigators normalize urine content results to creatinine [10]. Analyses have been performed on a yearly basis for the last 6 years and a statistical change will be reported to the PI and/or steering groups of the different projects.

We have also conducted an evaluation of changes in sodium and creatinine concentrations during repeated freeze-thaw cycles. Results will be presented in a future manuscript.

\section{RNA}

In the Biobank, both adult and umbilical cord blood samples collected in Tempus ${ }^{\mathrm{TM}}$ Blood RNA Tubes (Applied Biosystems) are stored at $-80{ }^{\circ} \mathrm{C}$. These tubes contain stabilizing reagents that preserve RNA transcripts, which make it possible to isolate RNA even after long-term storage. An ongoing RNA quality program is being conducted at the Biobank, which includes evaluation of effects on RNA quality after longterm storage (years) of whole blood in Tempus tubes, transportation effects (time and temperature) and low blood volume effects (i.e. the tube is filled with less blood than optimal). The results will be presented in a future manuscript. Any statistical change shown in the long-term stability project will be reported to the PI and/or steering groups of the different projects.

The material collected in Tempus tubes is precious and irreplaceable, and only one tube from each donor has been collected. Once isolated, RNA can be rather unstable, and therefore it may be better to isolate RNA from only parts of the sample, leaving the rest of the blood in the tube for future processing [11,12]. To address this, we have conducted analyses of aliquots of blood samples collected in Tempus tubes. Parts of the total blood-volume have been used for RNA isolation and analyses, and the remaining blood has been refrozen at $-80{ }^{\circ} \mathrm{C}$ for long-term storage before RNA isolation and analyses. The results will be presented in a future manuscript.

\section{RESULTS AND DISCUSSION}

The biospecimen quality program in the NIPH Biobank, as well as the specimen sample handling and storage protocols, have been developed to fit each project's needs within their budgets and the principles of quality assurance and quality control. One of the challenges when collecting biological specimens in longitudinal studies is not knowing what analyses will be performed on the banked material. New knowledge of sample integrity and developments in sample analysis, or requests for other kinds of analysis, may intro- 
duce different demands for information regarding the banked samples and their quality. When implementing a QMS based upon the NS-EN ISO 9001:2008 standard with continuous improvement as a pillar, it follows that our biospecimen quality program and tools, as well as improvement of processes, methods and documentation, will be evaluated and developed further to meet the needs of new customers, knowledge and analyses.

The NIPH Biobank uses different tools to achieve improvement, and one of them actively uses feedback from customers to improve processes and to update information about the quality of the biospecimens. We conduct an annual customer survey with questions on both project administration and how the biological material met their demands and expectations. We also ask a selection of the laboratories that perform analyses on the banked specimens to report their experiences, including quality controls and measures. All measures, reports and other feedback are thoroughly evaluated before archived. All useful analytical information about single samples is transferred to our LIMS and errors and complaints are followed up closely.

When choosing biobank samples for a study, researchers should be able to choose samples based on pre-analytical factors in addition to clinical or environmental factors. A standard coding system for preanalytical factors called SPREC has recently been published [13]. The NIPH Biobank has not implemented this yet, but preanalytical variables such as sample type, type of primary container, pre- and post-centrifugation conditions, centrifugation parameters and storage conditions are either tracked in the LIMS or can be found in the SOPs describing sample collection and processing.

The content of the biospecimen quality program has changed from measuring DNA purity when the NIPH Biobank was established in 1999, to cover both long term stability programs and a broader range of DNA quality control tests. When starting a thorough DNA QC program we tested every $8^{\text {th }}$ sample for concentration, fragmentation and PCR success. The extended program has given us valuable knowledge regarding how the DNA has met our requirements. The monitoring of the DNA quality has given us important information regarding the isolation- and normalization process. It has, on some occasions, led to temporary stops of the procedures in the laboratory to allow for evaluation and testing of our protocols and performance of our instruments, which in turn has led to changes i.e. the frequency of supplier performed service on pipetting robots used for the normalization process has been changed from annual to twice per year.

The electrophoresis of genomic DNA showed a significant amount of whole genome DNA in all tested samples, and less than $3.5 \%$ of the samples contained traces of fragmented DNA. The PCR success rate has been $100 \%$. Based on our results we have reduced the number tested to every $12^{\text {th }}$ sample for concentration.
For the fragmentation and PCR success part, we have reduced the number even further due to our own results, but also based on feedback regarding the DNA quality from laboratories using the samples. The test frequencies chosen are not based on an existing test rule, but are based on the evaluation of the equipment used in the process, test results and economy in the projects. However, in the monitoring of the concentration measurement process, we use DNA with three known concentrations, 20, 100 and $300 \mathrm{ng} / \mu \mathrm{l}$, and the $1_{2 S} / 1_{3 S}$ rules [14] to detect problems.

The DNA in a biobank is most likely to be used in several disciplines, like genomics, genetics, epigenonimcs and epigenetics and different parameters should therefore be included in a quality control program. With our present program we cover important parameters for most of the different disciplines, and others will be implemented in the future. One additional parameter could be OD 260/230 ratio measurements, since this measurement, as the OD 260/280 ratio provides information about the purity of the isolated DNA [15]. In addition, some recommend using OD 320 to determine purity and turbidity of the DNA [12].

Storage can affect the quality of the samples, and the quality programs to follow long-term storage for urine, plasma and RNA provide the biobank management and researchers requesting material with useful information about the stability of the stored samples. The $\mathrm{MoBa}$ project has guidelines for biomaterial usage which also include specifications on how much plasma must remain in the Biobank until the children reach 18 years of age (Researchers guidelines; http://www.fhi. no/dokumenter/fe6f6d962e.pdf). Thus, it is important to have a quality control program to assess the stability for these samples over an 18 year period, and beyond to cover the lifetime of the study. This is particularly important since most published human plasma longterm studies have covered a timeline shorter than the expected storage time in the MoBa study. For the Tempus RNA tubes it is of outmost importance to follow the quality of the isolated RNA after long term storage, and if the program shows changes in analytical or functional RNA quality, action must be taken.

The results from the freeze-thaw part of the plasma program is presented elsewhere [9]. The conclusion, however, has influenced the format the NIPH Biobank uses for plasma storage. Earlier both 96 well plates and Matrix single tubes were used. The 96 well plate format involves some challenges, since thawing and retrieving one sample on a plate, results in having to thaw all other samples on the plate as well. Even if the results showed that many plasma components were stable up to 30 freeze thaw cycles [9], we decided to use single tube formats for plasma in the Biobank only, and to transfer plasma stored in microtitre plates to single tubes. This will help minimize the number of freeze-thaw cycles of the plasma significantly, and whenever freeze-thawing does occur, it is logged in our LIMS. 
We believe that through our quality management and biospecimen quality programs, the NIPH Biobank is providing researchers with a good foundation for use of the material collected. The biospecimen quality program ensures that the material collected, processed, stored and retrieved are high quality specimens which can be used to get reproducible research results.

\section{ACKNOWLEDGEMENTS}

The NIPH Biobank is supported by NIH/NIEHS (grant no. NO1-ES-85433), NIH/NINDS (grant no. 1 UO1 NS 047537 01), EU/EARNEST (grant no. 007036) and the Norwegian Research Council/FUGE (grant no. 151918/S10). We gratefully acknowledge Kjersti S. Rønningen, Helle M. Meltzer, Jane A. Hoppin, Susan V. Baker, Nur Duale, Gunnar Brunborg, Anita Haugan, Trine Skjerden and Per Magnus for their contribution to the quality program.

\section{REFERENCES}

1. Betsou F, Barnes R, Burke T, Coppola D, Desouza Y, Eliason J, et al. Human biospecimen research: experimental protocol and quality control tools. Cancer Epidemiol Biomarkers Prev 2009; 18: 1017-1025.

2. Bonini P, Plebani M, Ceriotti F, Rubboli F. Errors in laboratory medicine. Clin Chem 2002; 48: 691-698.

3. Holland NT, Pfleger L, Berger E, Ho A, Bastaki M. Molecular epidemiology biomarkers - sample collection and processing considerations. Toxicol Appl Pharmacol 2005; 206: 261-268.

4. Magnus P, Irgens LM, Haug K, Nystad W, Skjærven R, Stoltenberg C. Cohort profile: the Norwegian Mother and Child Cohort Study (MoBa). Int J Epidemiol 2006; 35: 1146-1150.

5. Rønningen KS, Paltiel L, Meltzer HM, Nordhagen R, Lie KK, Hovengen R, et al. The biobank of the Norwegian Mother and Child Cohort Study: a resource for the next 100 years. Eur J Epidemiol 2006; 21: 619-625.

6. Yuille M, Illig T, Hveem K, Schmitz G, Hansen J, Neumaier M, et al. Laboratory management of samples in biobanks: European consensus expert group report. Biopreserv Biobank 2010; 8: 65-69.

7. Brooks A. Preventing "Identity Theft" at a Biobank Through Analytical and Functional Quality Control. 2011. http: //www.amp.org/Webinars/documents/BrooksWebinarHandout-2011.pdf. Accessed 14 Jun 2011.

8. Ivarsson M, Carlson J. Extraction, quantitation, and evaluation of function DNA from various sample types. Methods Mol Biol 2011; 675: 261-277.

9. Paltiel L, Rønningen KS, Meltzer HM, Baker SV, Hoppin JA. Evaluation of Freeze Thaw Cycles on stored plasma in the Biobank of the Norwegian Mother and Child Cohort Study. Cell Preserv Technol 2008; 6: 223230.

10. Spierto FW, Hannon WH, Gunter EW, Smith SJ. Stability of urine creatinine. Clin Chim Acta 1997; 264: $227-$ 232.

11. Bustin SA, Benes V, Nolan T, Pfaffl MW. Quantitative real-time RT-PCR - a perspective. J Mol Endocrinol 2005; 34: 597-601.

12. Santella RM. Approaches to DNA/RNA extraction and whole genome amplification. Cancer Epidemiol Biomarkers Prev 2006; 15: 1585-1587.

13. Betsou F, Lehmann S, Ashton G, Barnes M, Benson EE, Coppola D, et al. Standard preanalytical coding for biospecimens: defining the sample PREanalytical code. Cancer Epidemiol Biomarkers Prev 2010; 19: 10041011.

14. Westgard JO. Basic QC Practices, 2nd edn. Madison: Westgard QC, Inc, 2002.

15. Riemann K, Adamzik M, Frauenrath S, Egensperger R, Schmid KW, Brockmeyer NH, et al. Comparison of manual and automated nucleic acid extraction from whole-blood samples. J Clin Lab Anal 2007; 21: 244-248. 